\title{
Intervenção em terapia ocupacional com um sujeito com doença de Parkinson
}

\author{
Gustavo Artur Monzeli ${ }^{a}$, Ana Carolina Toniolo ${ }^{\mathrm{b}}$, Daniel Marinho Cezar da Cruz \\ ${ }^{a}$ Universidade Federal do Espírito Santo - UFES, Vitória, ES, Brasil. \\ bAmbulatório de Saúde Mental Álcool e/ ou Outras Drogas, Piracicaba, SP, Brasil. \\ 'Universidade Federal de São Carlos - UFSCar, São Carlos, SP, Brasil.
}

Resumo: Introdução: A doença de Parkinson-DP tem acometido um número cada vez maior de indivíduos, comprometendo as suas capacidades funcionais de forma progressiva e irreversível, exigindo ações preventivas e reabilitadoras. Objetivo: Teve-se por objetivo relatar o efeito da intervenção terapêutica ocupacional com um idoso de 70 anos diagnosticado com DP. Método: Trata-se de um relato de experiência. Foi elaborado um plano de intervenção com um programa de exercícios para atividades extra-atendimento e visita domiciliar para observação de possíveis dificuldades e riscos no ambiente. A mensuração da independência foi realizada por meio da Medida de Independência Funcional-MIF. Essa ocorreu durante o período de seis meses. Resultados: Identificou-se melhora de desempenho para as atividades de alimentação e escrita. Na MIF houve aumento de um ponto. Conclusão: Destaca-se a importância da intervenção da terapia ocupacional, que neste contexto favoreceu a melhora do desempenho desse sujeito em suas Atividades de Vida Diária, contribuindo para a manutenção das capacidades funcionais.

Palavras-chave: Terapia Ocupacional, Doença de Parkinson, Reabilitação, Atividades Cotidianas.

\section{Occupational therapy intervention in a subject with Parkinson disease}

\begin{abstract}
Introduction: Parkinson disease-PD has affected an increasing number of individuals, compromising their functional skills progressively and irreversibly, requiring preventive and rehabilitative actions. Objective: To present and discuss the effect of occupational therapy intervention with a 70-year old patient diagnosed with PD. Method: This is an experience report with a single subject. An intervention plan including an exercise program for extra-care activities and home visits for observation of possible difficulties and risk of falls in the environment has been prepared. The measurement of independence was performed using the Functional Independence Measure-FIM. The intervention occurred during six months. Results: It was identified improvement in eating and writing activities. There was one score of improvement documented in FIM. Conclusion: The importance of the intervention of occupational therapy is enforced, which in this context improved the occupational performance of this subject in the daily life activities, contributing for maintaining functional performance.
\end{abstract}

Keywords: Occupational Therapy, Parkinson Disease, Rehabilitation, Activity of Daily Living.

Autor para correspondência: Gustavo Artur Monzeli, Departamento de Terapia Ocupacional, Universidade Federal do Espírito Santo, Avenida Marechal Campos, 1468, Maruípe, CEP 29043-900, Vitória, ES, Brasil, e-mail: gustavo.monzeli@gmail.com

Recebido em Dez. 20, 2014; $1^{\text {a }}$ Revisão em Fev. 3, 2015; 2a Revisão em Jul. 6, 2015; Aceito em Out. $25,2015$. 


\section{Introdução}

A Doença de Parkinson (DP) é uma desordem de movimento crônica, progressiva e sua causa ainda é desconhecida. Embora no momento não haja cura, existem opçóes de tratamento, como medicação e cirurgia para gerenciar seus sintomas (PARKINSON..., 2015). Mais de um milhão de americanos vivem com a DP, número maior do que o combinado de pessoas diagnosticadas com esclerose múltipla, distrofia muscular e a doença de Lou Gehrig, conhecida também como a Esclerose Lateral Amiotrófica (PARKINSON..., 2015). Estima-se que sete a 10 milhóes de pessoas no mundo convivem com a DP. No Brasil, não são encontrados dados a respeito até o presente momento.

A DP ocorre devido à degeneraçáo de neurônios dopaminérgicos da substância negra e do lócus ceruleus, o que acarreta uma diminuição na quantidade de dopamina para o corpo estriado (MENESES; TEIVE, 1996). Como consequência, a pessoa apresenta prejuízos, principalmente, nas funçôes motoras. Ocorre uma perda de $60 \%$ dos neurônios e surgem os chamados sinais cardinais: rigidez, bradicinesia, tremor de repouso e alteraçóes posturais (MENESES; TEIVE, 1996; GONÇALVES; ALVAREZ; ARRUDA, 2007).

Hoehn e Yahr (1967) propuseram a DP a partir da gravidade dos comprometimentos em uma escala. A versáo modificada dessa escala divide-se em 7 estágios (LANA et al., 2007). Os estágios encontram-se no Tabela 1 a seguir.

Há uma variedade de tratamentos medicamentosos e outras terapias disponíveis para melhorar a qualidade de vida e o engajamento social de pessoas que têm essa doença. Esses medicamentos precisam de administração e atenção cuidadosa em termos de dose e horário (LINDAHL, 2011). Quanto ao tratamento não medicamentoso, a fisioterapia e a terapia ocupacional têm papel essencial, principalmente nos pacientes com distúrbio acentuado do equilíbrio e da marcha (FERRAZ, 1999). O comprometimento motor e as limitaçóes funcionais são classicamente sequelas reconhecidas, sendo a terapia ocupacional um complemento importante para a gestão farmacológica (RAO, 2010).

\subsection{Doença de Parkinson e a intervenção da terapia ocupacional}

A intervenção da terapia ocupacional baseia-se em amenizar os efeitos da doença sobre a vida funcional e/ou psicossocial desses indivíduos, tendo como um dos focos o impacto sobre as Atividades da Vida Diária- AVD. A DP pode acarretar prejuízos físicos e funcionais devido à alteração na força muscular, coordenação, amplitude e intervalo de movimentos. Tais disfunções, de acordo com cada estágio, podem ser evidenciadas progressivamente em algumas AVD como alimentação e autocuidado, bem como as relacionadas aos componentes da coordenação motora fina (punho e dedos), por exemplo, para a atividade de escrita manual.

O terapeuta ocupacional pode utilizar dispositivos tecnológicos, sugerir mudanças ambientais e facilitar a execução de tarefas diversas tornando-as mais efetivas, seguras, econômicas, confortáveis e prazerosas (ALMEIDA; CRUZ, 2009). Dixon et al. (2007) relatam em sua pesquisa que mesmo com os tratamentos cirúrgicos e terapias medicamentosas, os pacientes com DP desenvolveráo deficiências progressivas. A sintomatologia motora e não motora e sua interaçáo com o ambiente pode ser muito complexa. Segundo esses autores, o papel do terapeuta ocupacional reside em auxiliar o paciente a manter seu usual nível de autocuidado, trabalho e lazer ao máximo de tempo possível. Quando isso não é mais possível, terapeutas ocupacionais devem ajudar na adaptação do ambiente físico e social, no

Tabela 1. Escala de Hoehn e Yahr Modificada.

\begin{tabular}{ll}
\hline \multicolumn{1}{c}{ ESTÁGIO } & \\
\hline Estágio 0 & Nenhum sinal da doença \\
Estágio 1 & Doença unilateral \\
Estágio 1,5 & Envolvimento unilateral e axial \\
Estágio 2 & Doença bilateral sem déficit de equilíbrio \\
Estágio 2,5 & Doença bilateral leve, com recuperação no "teste do empurrão" \\
Estágio 3 & Doença bilateral leve a moderada; alguma instabilidade postural; capacidade para viver \\
& independente \\
Estágio 4 & Incapacidade grave, ainda capaz de caminhar ou permanecer de pé sem ajuda \\
Estágio 5 & Confinado à cama ou cadeira de rodas a não ser que receba ajuda \\
\hline
\end{tabular}

Fonte: Shenkman et al. (2001). 
desenvolvimento e valorização de novos papéis e atividades (DIXON et al., 2007).

Terapeutas ocupacionais devem também agregar valor em sua intervenção não somente aos indivíduos com DP, os quais encontram a sua vida diária comprometida, mas também com os seus cuidadores imediatos. Segundo esses autores, evidências sobre intervençôes nessa direção são inconclusivas, assim como carecem de estudos rigorosos, o que destaca a necessidade de produção de evidências e documentação da prática dos terapeutas ocupacionais (STURKENBOOM et al., 2013a).

Diante das atuais mudanças na área da saúde, ser capaz de obter e utilizar evidências de pesquisa para sustentar o tratamento de terapia ocupacional tornou-se cada vez mais importante (MURPHY; TICKLE-DEGNEN, 2001). Embora exista uma dificuldade em se comprovar os efeitos de intervençóes da terapia ocupacional na DP (STURKENBOOM et al., 2013a, 2013b, 2014; RAO, 2010; DIXON et al., 2007; GAUDET, 2002), uma breve síntese sobre a intervenção da terapia ocupacional junto a indivíduos com DP permite compreender os tipos de intervençáo realizados e os seus efeitos.

A pesquisa de revisão de Dixon et al. (2007) teve por objetivos comparar a eficácia e efetividade da terapia ocupacional com grupo placebo ou grupo controle em pacientes com DP. Apenas estudos randomizados e controlados foram selecionados. Os autores encontraram dois estudos com 84 pacientes no total. Embora ambos os estudos tenham reportado um efeito positivo da intervenção, todos os ganhos foram pequenos. Os estudos não tiveram tratamento placebo adequado e usaram um número pequeno de pacientes. O método de randomização e alocação dos sujeitos também não foi especificado em um desses estudos. Os autores concluíram que não havia, até aquele momento, evidência suficiente para comprovar a eficácia da terapia ocupacional no tratamento de pessoas com DP (DIXON et al., 2007).

Posteriormente, Ma et al. (2009) investigaram o efeito do peso de um utensílio para alimentação no movimento do membro superior em pessoas com DP a partir de um estudo controlado. Foram submetidos dezoito adultos com e sem DP, pareados por idade. Cada participante realizou uma tarefa de transferência de comida usando colheres de três pesos diferentes: leve ( $35 \mathrm{~g})$; controle $(85 \mathrm{~g})$; e pesados $(135 \mathrm{~g})$. As variáveis cinemáticas do movimento do braço foram obtidas e comparadas entre as condiçóes. As variáveis cinemáticas do movimento do braço incluíram o tempo de movimento, a velocidade de pico e o número de unidades de movimento. Como principais resultados, identificou-se que o peso do utensílio afetou significativamente o movimento em todos os participantes. Ambos os grupos tinham menos unidades de movimento na condição leve (grupo DP: 22.18, controles: 19,89) do que na condição pesada (grupo DP: 22.68, controles: $21,36)$, sugerindo o movimento mais suave na primeira condição. Além disso, ambos os grupos apresentaram maior pico de velocidade na condição leve do que na condição pesada. A conclusão do estudo implica um utensílio leve que pode facilitar o movimento mais suave e com maior velocidade do braço em pessoas com DP, favorecendo a AVD de alimentação (MA et al., 2009).

O estudo transversal de Nickel et al. (2010) abrangeu a avaliação do desempenho ocupacional de 46 sujeitos com DP por meio da Medida de Desempenho Ocupacional Canadense (COPM) e domínios da Classificação Internacional de Funcionalidade, Incapacidade e Saúde- CIF. A pesquisa identificou que as atividades mais comprometidas foram as da vida comunitária, social e cívica $(32,6 \%)$; a mobilidade $(26,1 \%)$; o cuidado pessoal (27,1\%); a vida doméstica (10,9\%); e a aprendizagem e aplicação de conhecimento (8,7\%). Também se identificou correlação média da variável rigidez com os cinco domínios classificados na CIF $(\mathrm{p}<0,01)$. Os pesquisadores recomendam a promoção e manutenção da vida comunitária, social e cívica para sujeitos com DP e a rigidez como componente importante para o desempenho ocupacional (NICKEL et al., 2010).

A pesquisa de Sturkenboom et al. (2013b) teve por objetivo avaliar a viabilidade de um estudo randomizado controlado e o potencial impacto da terapia ocupacional na DP. O processo e os resultados foram quantitativa e qualitativamente avaliados num estudo multicêntrico exploratório. Participaram quarenta e três pacientes residentes na comunidade e que apresentavam dificuldades nas AVD. O grupo de intervenção constou de dez semanas de terapia ocupacional em domicílio, de acordo com as diretrizes holandesas de terapia ocupacional para a DP. O grupo controle não teve nenhuma intervenção da terapia ocupacional. Foi utilizada a Medida Canadense de Desempenho Ocupacional (COPM) e a avaliação de habilidades motoras e do processo. As medidas de desfecho revelaram-se insignificantes para pequenos efeitos em favor do grupo de intervenção, embora quase todos os pacientes e acompanhantes do grupo de intervenção apresentassem satisfação com os resultados (STURKENBOOM et al., 2013b).

Não obstante os resultados das pesquisas existentes fossem de difícil comprovação, há evidências que 
sugerem que o tratamento da terapia ocupacional produz melhorias na qualidade de vida e no comportamento motor de pessoas com DP (RAO, 2010).

Em pesquisa mais recente, avaliou-se a eficácia da terapia ocupacional em melhorar as AVD de pacientes com DP (STURKENBOOM et al., 2014). O método foi um estudo multicêntrico, um ensaio clínico randomizado controlado em dez hospitais, em nove redes regionais holandesas (ParkinsonNet), com avaliação aos 3 meses e 6 meses. Pacientes com DP que relataram dificuldades em AVD foram incluídos na amostra, juntamente com os seus cuidadores primários. Os pacientes foram distribuídos aleatoriamente (2: 1) para o grupo de controle ou intervenção por um algoritmo de minimização gerado por computador. A intervenção consistiu de 10 semanas de terapia ocupacional em domicílio; indivíduos do grupo controle receberam cuidados habituais, sem terapia ocupacional. O desfecho primário foi o desempenho autopercebido nas atividades diárias em 3 meses, avaliado pela Medida Canadense de Desempenho Ocupacional (COPM). No início do estudo, a pontuação média na COPM foi $4 \cdot 3$ (IQR 3 · 5-5 · 0) no grupo de intervenção e $4 \cdot 4(3 \cdot 8-5 \cdot 0)$ no grupo de controle. Aos 3 meses, esses índices foram de $5 \cdot 8(5 \cdot 0-6 \cdot 4)$ e $4 \cdot 6$ (4 - 6-6 6 6), respectivamente. A diferença média ajustada na pontuação entre os grupos aos 3 meses foi a favor do grupo de intervenção $(1 \cdot 2 ; 95 \% \mathrm{CI}$ $0 \cdot 8-1 \cdot 6 ; \mathrm{p}<0 \cdot 0001)$. Concluiu-se que a terapia ocupacional individualizada em domicílio levou a uma melhoria no desempenho autopercebido nas AVD em pacientes com DP (STURKENBOOM et al., 2014).

Em revisão sistemática também recente sobre as intervençôes da terapia ocupacional em pacientes com DP, três categorias foram analisadas: 1) exercício e atividade física; 2) pistas ambientais, estímulos e objetos; e 3) autogestão e estratégias cognitivo-comportamentais. Evidência de forte a moderada foi encontrada para tarefas específicas de treinamento de atividades físicas sobre o desempenho motor, estabilidade postural e equilíbrio. Baixa a moderada evidência indica que atividades mais complexas e atividades multimodais auxiliam na melhoria de movimento em atividades funcionais. Evidência moderada foi encontrada quanto ao uso de suportes externos durante a mobilidade funcional ou outras atividades que exigem movimento, tendo efeito positivo no controle motor (FOSTER; BEDEKAR; TICKLE-DEGNEN, 2014).

Nessa direção, a documentação da prática da terapia ocupacional com essa populaçáo a partir de evidências faz-se essencial para o desenvolvimento da profissão e de intervençôes inovadoras que possam beneficiar esses sujeitos. No Brasil, ainda são escassos os estudos publicados sobre terapia ocupacional na intervenção com pacientes com DP. Logo, tem-se por objetivo relatar a experiência de intervençáo da terapia ocupacional ao longo de seis meses com um idoso de 70 anos diagnosticado com DP, bem como demonstrar o efeito dessa intervenção na independência funcional para as AVD.

\section{$2 \mathrm{O}$ relato da experiência}

Segundo Nunes (2008), os casos clínicos são também recursos didáticos que favorecem a aquisição de conhecimento e a formação profissional e isto é possível a partir da análise dos resultados de avaliaçôes funcionais em diferentes disfunçôes neurológicas.

Trata-se de um relato de experiência que reporta a intervenção com um sujeito com Doença de Parkinson. Esses atendimentos fizeram parte do estágio supervisionado em terapia ocupacional, em que participaram um docente e dois alunos. Cabe destacar que os dados a serem apresentados foram obtidos a partir das informaçóes documentadas ao longo do processo terapêutico, tais como imagens e avaliaçôes funcionais. Em funçáo da instituição em que o sujeito foi atendido já apresentar procedimentos éticos, tais dados já são protegidos pela assinatura de um Termo de Consentimento institucional. Além disto, foram garantidos todos os procedimentos para não identificação do sujeito, como a alteração de informaçóes pessoais que pudessem identificá-lo.

\subsection{O processo da terapia ocupacional: avaliação, intervenção e resultados}

Para melhor compreensão, estruturou-se a apresentação do caso, a partir do processo da terapia ocupacional, definido como a Avaliação, a Intervenção e os Resultados (AMERICAN..., 2008).

\subsection{Avaliações}

No ano de 2009, o sujeito foi encaminhado por um neurologista com o diagnóstico de DP (estágio 1) para o serviço de Terapia Ocupacional de uma Unidade de Reabilitação pública de uma cidade do interior do Estado de São Paulo.

A queixa inicial do sujeito era a de "tremedeira na mão direita”, mão que lhe era mais funcional pelo fato de ser destro. O sujeito relatou que os sintomas haviam se manifestado há cerca de um ano e meio (tempo referente à ocasião do início 
dos atendimentos). Depois do ingresso do sujeito no referido serviço, este foi atendido durante seis meses (22/5/2009 a 30/11/2009), totalizando 16 atendimentos, na frequência de uma vez por semana. Neste período, este era acompanhado por uma equipe composta por psicólogo, fisioterapeuta e terapeuta ocupacional.

\subsection{Anamnese}

E., do sexo masculino, encontrava-se com 70 anos. Fazia acompanhamento medicamentoso com o neurologista que o encaminhou ao serviço. O sujeito era natural de uma cidade do interior do Estado de São Paulo, católico, casado há 48 anos e residia com esposa, um filho, duas filhas e três netos. A família era composta por seis filhos, 14 netos e 17 bisnetos.

Em relação às ocupaçôes produtivas de estudo e trabalho, respectivamente, o sujeito cursou até a $4^{a}$ série do ensino fundamental e trabalhou lavando carros em postos de gasolina por 52 anos; sendo aposentado há 13 anos. Além disso, foi um instrumentista registrado pela Associação Brasileira dos Músicos. No momento da anamnese, o sujeito relatou desempenhar poucas atividades restringindo-se a assistir TV com a esposa e os netos todas as noites. $\mathrm{O}$ sujeito não possuía histórico de tabagismo ou etilismo. Quanto às atividades de descanso e dormir, não foi relatada queixa relacionada ao sono.

\subsection{Avaliação funcional}

Nas avaliaçôes iniciais para a intervenção no caso, preconizou-se pela escolha de uma avaliação de independência em AVD, além das avaliaçôes clínicas institucionais e dados registrados em prontuário pela equipe que atuava no caso. Com o objetivo de avaliar o grau de dependência para realizar as AVD, aplicou-se na pré e pós-intervenção a Medida de Independência Funcional- MIF, validada no Brasil (RIBERTO et al., 2004).

\subsection{Intervenção em terapia ocupacional}

O sujeito participou de dezesseis sessões de terapia individual e também foi inserido nas atividades de um grupo de socialização musical para idosos, o qual ocorria nas mesmas dependências do local de atendimento. Esse grupo, entretanto, não foi o foco deste relato.

Os atendimentos foram organizados de acordo com as necessidades identificadas a partir de ampla avaliação e na história de vida do sujeito.
Essas atividades abrangeram os seguintes procedimentos: a) programa de exercícios físicos; b) estimulação da independência em AVD; c) visita domiciliar para a adaptação do ambiente; e d) orientaçóes para a realizaçáo de atividades em domicílio, descritas brevemente a seguir:

a) Programa de Exercícios Físicos: o programa de exercícios físicos abrangeu o enfoque na funcionalidade dos membros superiores e no tronco enquanto componente importante para fornecer estabilidade para a função das mãos. Os exercícios foram realizados ao longo de todos os atendimentos, priorizando-se o "input" sensorial tátil e proprioceptivo durante a funçáo dos membros superiores em diferentes posturas (decúbitos, sedestação e ortostase) e estimulando os componentes motores de alcance, preensão, manipulação e soltar de objetos com variados tamanhos, formas, funçóes, pesos e texturas, uni e bilaterais. A coordenação motora fina também foi estimulada a partir de objetos pequenos e de atividades funcionais que requeriam preensóes como a palmar, pinça ponta a ponta, pinça polpa a polpa, dentre outras. Precedeu a todos os exercícios, autoalongamentos e alongamentos passivos das musculaturas envolvidas na função, tais como trapézio fibras superiores, deltoide fibras médias e anteriores, peitoral maior e menor, bíceps braquial, musculaturas de punho e mão.

b) Estimulação da independência em AVD: para as AVD, a intenção foi a de ensinar estratégias compensatórias e uso de dispositivos de tecnologia assistiva, quando necessário. Em função do paciente ser independente na maioria dos itens avaliados pela MIF, foram enfocadas as atividades de alimentação, autocuidado e mobilidade funcional tais como a atividade de rolar no leito, sentar e levantar da cama, dentre outras.

c) Visita domiciliar para a adaptaçáo do ambiente: foi feita uma visita domiciliar com o objetivo de redução de riscos de quedas nesse ambiente.

d) Orientaçóes para a realizaçáo de atividades em domicílio: adotou-se um caderno de caligrafia para a realização de tarefas semanais de escrita manual e acompanhamento do quadro de micrografia. 
As atividades desenvolvidas ocorreram em função das principais queixas relatadas ao longo dos atendimentos: o tremor unilateral de membro superior direito, a micrografia (na atividade de escrita) e a dificuldade em tocar instrumentos musicais, no caso o trombone de vara, habilidade esta que o sujeito aprendeu aos 20 anos de idade.

Baseado em tais relatos e observações, foi elaborado um Programa de Exercícios Semanais para o sujeito realizar em casa. As orientaçôes foram feitas por escrito e o sujeito poderia relatar sensaçóes e/ou dificuldades percebidas durante cada atividade.

Em dado momento do tratamento foram inseridas atividades de coordenaçáo motora fina, incluindo exercícios com rotação de tronco e envolvendo diferentes preensóes (Figura 1). Iniciou-se, assim, a estimulaçáo da atividade de escrita (devido à micrografia), realizados em um caderno de caligrafia (Figura 2) e alongamentos, por exemplo, da musculatura intrínseca da mão (Figura 3).

\subsection{Os resultados da intervenção}

A partir do uso da MIF, se identificou que o sujeito desempenhava a maioria das atividades com independência completa (com tempo adequado, com segurança e sem adaptaçóes), obtendo pontuação 7. O escore total na MIF, para a avaliaçáo inicial, foi de 120 pontos, para um total máximo de 126 pontos, sendo classificado como independente. A despeito dessa classificação, alguns itens apresentaram pontuação abaixo de 7 , com algum grau de modificação ou ajuda física. Esses itens encontram-se listados no Tabela 2 a seguir.

As figuras 4 e 5 a seguir ilustram os resultados de pré e pós intervenção para o caso apresentado, a partir da Medida de Independência Funcional:

As figuras anteriores devem ser compreendidos em relação ao fato de que, quanto mais aberto o círculo em relaçáo ao seu centro, maior a independência do sujeito. Por meio das figuras 4 e 5 , observa-se um aumento da independência referente à atividade de alimentaçáo,

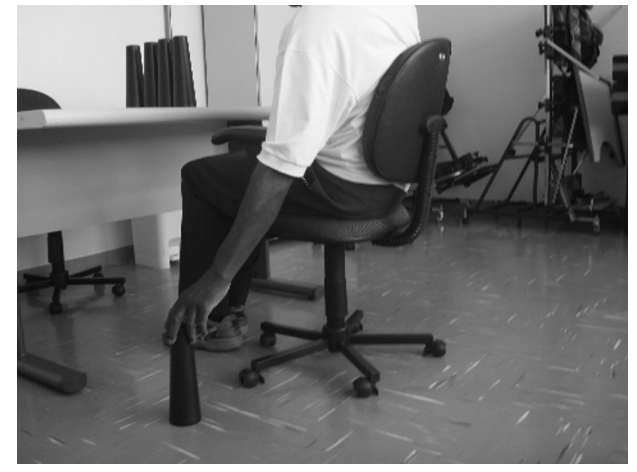

Figura 1. Exercícios de preensões.

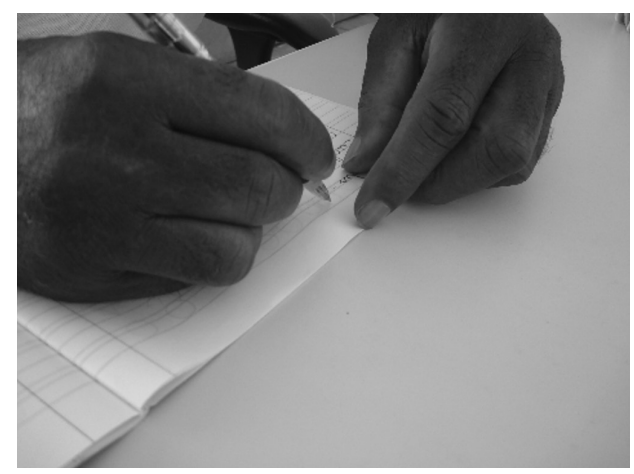

Figura 2. Exercício de escrita manual.

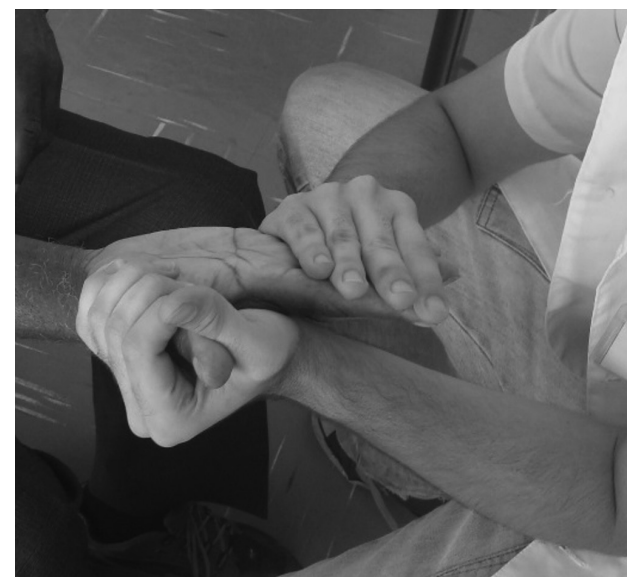

Figura 3. Alongamento da musculatura intrínseca da mão.

Tabela 2. Pontuação do sujeito na Medida de Independência Funcional- MIF.

\begin{tabular}{lll}
\hline \multicolumn{1}{c}{ Itens } & \multicolumn{1}{c}{ Pontuação } & \multicolumn{1}{c}{ Limitações/ Restrições } \\
A. Comer & $\begin{array}{c}\text { 6(Independência Modificada, pelo } \\
\text { uso de dispositivo de tecnologia } \\
\text { assistiva) }\end{array}$ & $\begin{array}{l}\text { Lentificação; uso exclusivo de colher às } \\
\text { refeições }\end{array}$ \\
$\begin{array}{l}\text { B. Cuidados com a } \\
\text { aparência }\end{array}$ & $\begin{array}{c}\text { (Assistência Mínima, Paciente }= \\
75 \%+)\end{array}$ & $\begin{array}{l}\text { Dependência total para a tarefa de fazer a } \\
\text { barba }\end{array}$ \\
\hline Andar & $\begin{array}{c}\text { (Independência Modificada, pelo } \\
\text { uso de meio auxiliar de locomoção) }\end{array}$ & $\begin{array}{l}\text { Lentificação durante a marcha } \\
\text { Escadas }\end{array}$ \\
$\begin{array}{c}\text { 6(Independência Modificada, pelo } \\
\text { uso do corrimão) }\end{array}$ & Lentificação ao subir e ao descer \\
\hline
\end{tabular}




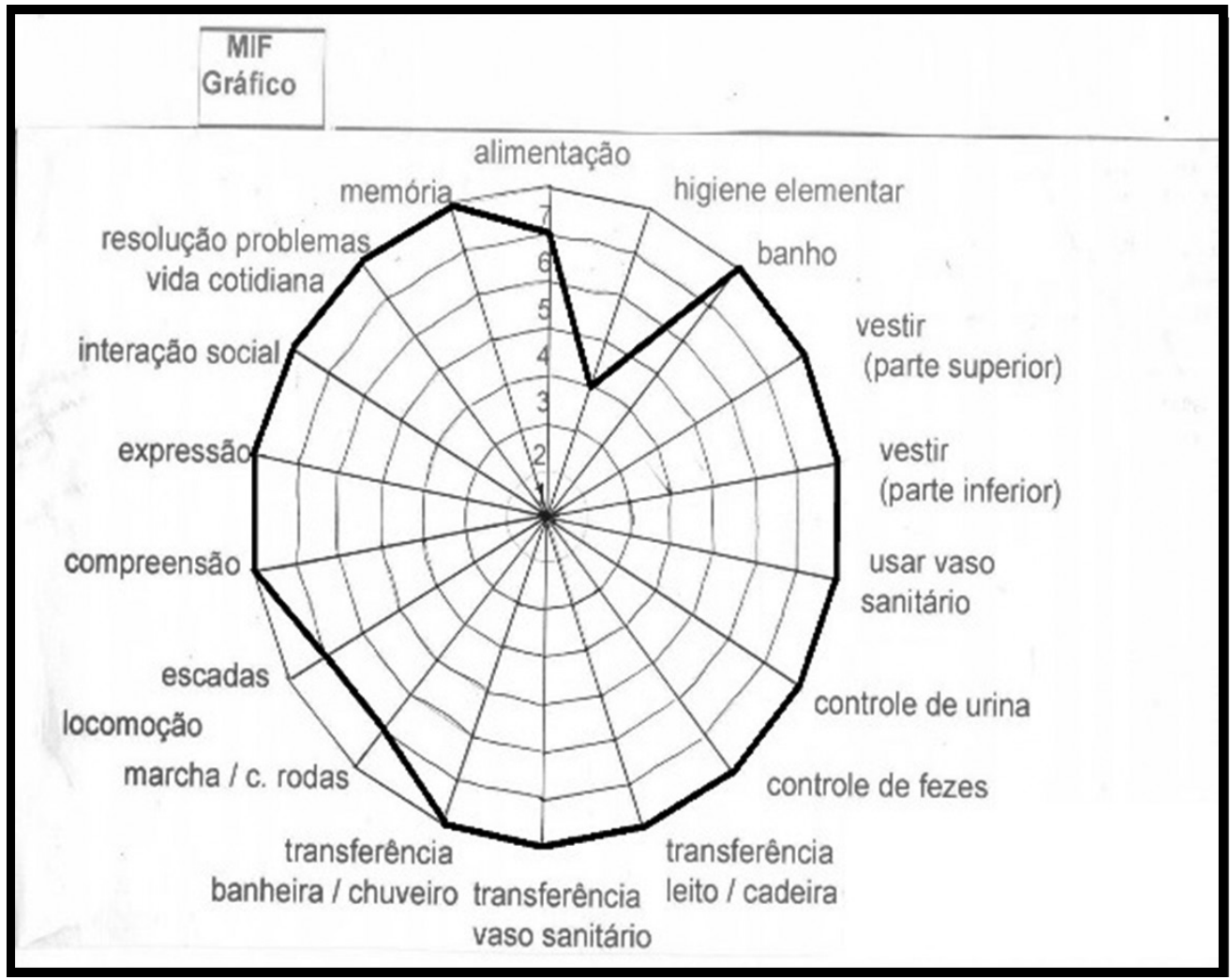

Figura 4. Pré-intervenção.

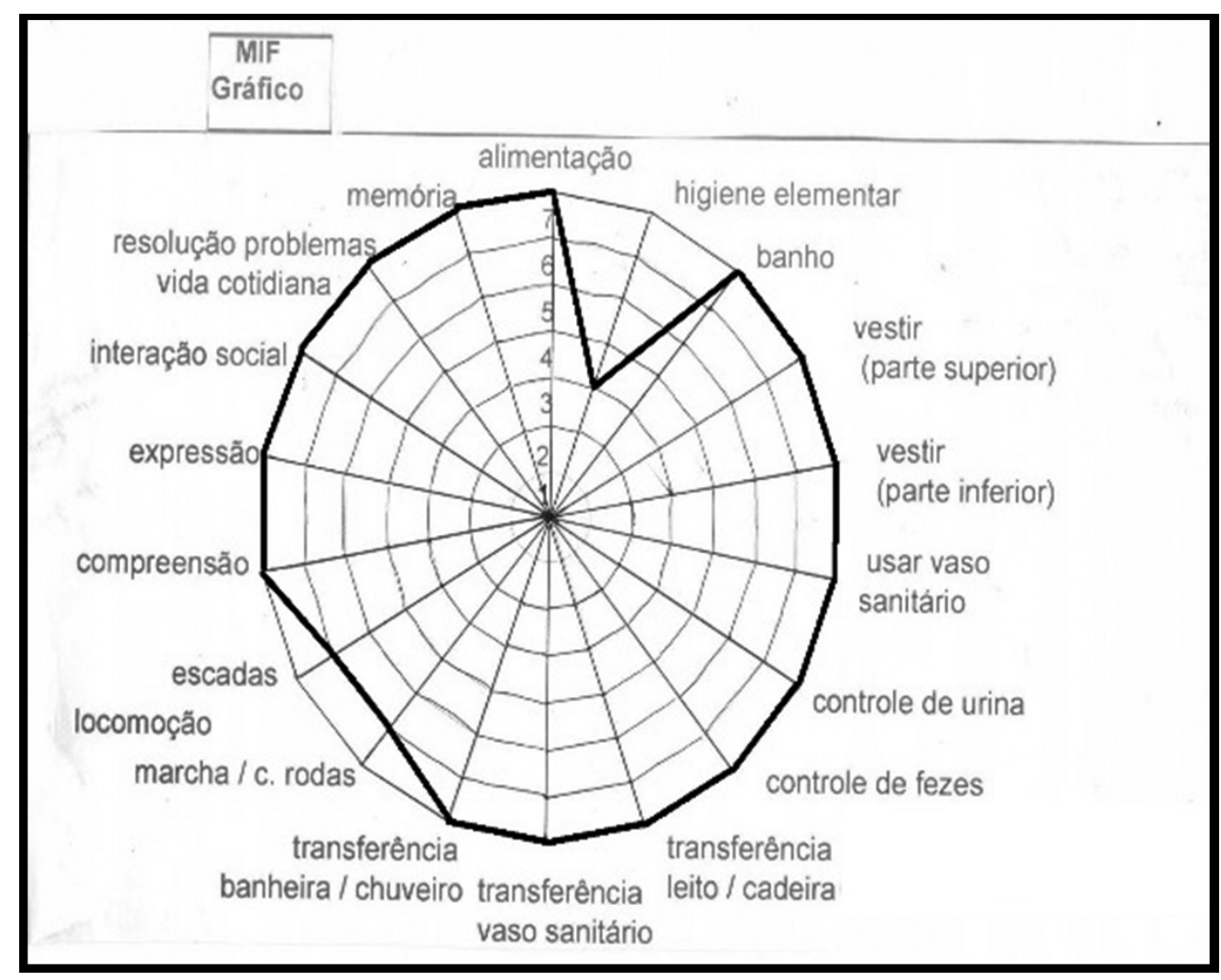

Figura 5. Pós-intervenção. 
que, na avaliação inicial, recebeu pontuação 6 e, ao final, passou para 7, demonstrando independência completa. Acredita-se que a independência completa para o item de alimentaçáo foi conquistada pelo foco nos exercícios e atividades que trabalhavam questôes como estimulação sensorial coordenação motora fina, o que também refletiu na amenização da micrografia e na melhora da destreza manual como um todo. Esses achados reforçam a importância da atividade física, reportada em estudos anteriores (RAO, 2010; RANSMAYR, 2011).

Nesse sentido, é factível que a intervenção em terapia ocupacional ao enfocar a terapia centrada em AVD pode manter capacidades ou mesmo aumentar a independência a partir de diferentes estratégias de ação conforme apontam as pesquisas mais recentes (STURKENBOOM et al., 2014; FOSTER; BEDEKAR; TICKLE-DEGNEN, 2014).

Observa-se também nas figuras 4 e 5 que todas as áreas afetadas pela DP correspondem ao domínio motor da MIF, a saber: escadas, marcha, higiene elementar (especificamente o fazer a barba). Esse item não sofreu modificaçôes, embora tenham sido feitas orientaçôes e proposições de adaptaçôes. Atribui-se a não evolução nesse item, em parte, ao fato de a rigidez possivelmente ser o componente que mais dificultou a atividade de se barbear, por exigir movimentos contínuos e de destreza e coordenação motora grossa (ombro) do membro superior. O estudo de Nickel et al. (2010) reforça esse achado, na medida em a pesquisa de fato identificou que a rigidez influenciou a maior parte das atividades funcionais desempenhadas pelos indivíduos com DP.

Porém, mesmo ao se considerar a DP como uma doença progressiva, acredita-se que a intervenção auxiliou na manutenção da capacidade funcional do sujeito ao longo de seis meses, não se observando, por exemplo, piora relativa a esse aspecto. A MIF foi uma medida objetiva importante para sustentar essa afirmação, uma vez que não foi reportada involução motora durante esse período.

Qualitativamente, também pode se destacar que, através da comparação entre as primeiras e as últimas atividades realizadas no caderno de caligrafia, foi notada visível melhora do quadro de micrografia. Isso em parte se atribuiu aos exercícios para melhorar a destreza manual. Acredita-se que os exercícios foram importantes para auxiliar o sujeito na realização das atividades que exigiam destreza fina. Embora haja insuficiência de evidências para tratamento de reabilitação na DP, pesquisas de alto nível, como os estudos controlados, recentemente têm demonstrado a melhoria da mobilidade e equilíbrio após o treinamento de força e resistência muscular, controle de tronco, e amplitude e ritmicidade dos movimentos nesses sujeitos (RANSMAYR, 2011). Além disso, as estratégias de atenção e cognitivas têm favorecido a consciência corporal e os movimentos sequenciados. Recursos como a dança, pistas sensoriais (auditiva, visual, tátil) e cognitivas têm sido eficazes para problemas de marcha e de equilíbrio, por exemplo (RANSMAYR, 2011).

Para dar sequência às atividades desenvolvidas em ambiente terapêutico também foi produzida uma cartilha de orientação para exercícios e autoalongamentos. Além dos atendimentos em setting terapêutico, foi realizada uma visita à residência do paciente e aplicado um roteiro de visita domiciliar. Observou-se que algumas mudanças eram necessárias, tais como: eliminação de tapetes e do "criado mudo" ao lado da cama, adaptaçáo de interruptores de luz e colocação de um corrimão na escada de acesso à casa. O paciente e a família foram orientados sobre as mudanças no domicílio, que poderiam contribuir para maior segurança e melhor desempenho do sujeito em suas atividades.

$\mathrm{Na}$ visita domiciliar de retorno à residência, constatou-se que a família aderiu às sugestóes de adequaçáo do ambiente quanto à retirada de tapetes que ofereciam risco de queda, bem como à instalação de um corrimão nas escadas de acesso à casa. Colaborando assim com a proposta de métodos que simplificassem a realização de tarefas diárias no sentido de melhorar a efetividade na realização dessas tarefas, ajudando na superação dos efeitos sintomáticos da doença no cotidiano (ALMEIDA; CRUZ, 2009).

\section{Conclusão e recomendações}

Para o relato de experiência apresentado, a intervenção da terapia ocupacional promoveu a manutenção da capacidade funcional do sujeito ao longo de seis meses, não havendo deterioração de funçóes, mas a melhora da funcionalidade, particularmente, para as atividades de alimentação e escrita manual.

Enfatiza-se a importância do processo terapêutico-ocupacional que neste contexto contribuiu para a minimização dos sintomas da DP e o impacto destes nas atividades cotidianas do sujeito. Recomenda-se a documentação constante da prática da terapia ocupacional com esses sujeitos a fim de promover identificação dos diferentes efeitos das intervençôes nas diversas fases da doença. 


\section{Referências}

ALMEIDA, M. H. M.; CRUZ, G. A. Intervençōes de terapeutas. Revista de Terapia Ocupacional da USP, São Paulo, v. 20, n. 1, p. 29-35, 2009.

AMERICAN OCCUPATIONAL THERAPY ASSOCIATION - AOTA. Occupational therapy practice framework: domain \& process. The American Journal of Occupational Therapy, Boston, v. 62, n. 6, p. 625683, 2008. http://dx.doi.org/10.5014/ajot.62.6.625. PMid:19024744.

DIXON, L. et al. Occupational therapy for patients with Parkinson's disease. Cochrane Database Systematic Reviews, Oxford, v. 18, n. 3, p. 1-3, 2007. PMid:17636709.

FERRAZ, H. B. Tratamento da doença de Parkinson. Revista Neurociências, Rio de Janeiro, v. 7, n. 1, p. 6-12, 1999.

FOSTER, E. R.; BEDEKAR, M.; TICKLE-DEGNEN, L. Systematic review of the effectiveness of occupational therapy-related interventions for people with Parkinson's disease. American Journal Occupational Therapy, Bethesda, v. 68, n. 1, p. 39-49, 2014. http://dx.doi. org/10.5014/ajot.2014.008706. PMid:24367954.

GAUDET, P. Measuring the impact of Parkinson's disease: an occupational therapy perspective. Canadian Jounal Occupational Therapy, Ottowa, v. 69, n. 2, p. 104-113, 2002. http://dx.doi. org/10.1177/000841740206900206. PMid:11977869.

GONÇALVES, L. H. T.; ALVAREZ, A. M.; ARRUDA, M. C. Pacientes portadores da doença de Parkinson: significado de suas vivências. Acta Paulista de Enfermagem, São Paulo, v. 20, n. 1, p. 62-68, 2007.

HOEHN, M.; YAHR, M. D. Parkinsonism: onset, progression and mortality. Neurology, Minneapolis, v. 17, n. 5, p. 427-442, 1967. http://dx.doi.org/10.1212/ WNL.17.5.427. PMid:6067254.

LANA, R. C. et al. Percepção da qualidade de vida de indivíduos com doença de parkinson através do PDQ39. Revista Brasileira de Fisioterapia, São Carlos, v. 11, n. 5, p. 397-402, 2007. http://dx.doi.org/10.1590/s141335552007000500011 .

LINDAHL, D. A. Parkinson's: treating the symptoms. British Jounal Nursing, London, v. 20, n. 14, p. 852-857, 2011.

MA, H. I. et al. The effect of eating utensil weight on functional arm movement in people with Parkinson's disease: a controlled clinical trial. Clinical Rehabilitation, London, v. 23, n. 12, p. 1086-1092, 2009. http://dx.doi. org/10.1177/0269215509342334. PMid:19906764.

MENESES, M. S.; TEIVE, H. A. G. Histórico. In: MENESES, M. S.; TEIVE, H. A. G. Doença de Parkinson: aspectos clínicos e cirúrgicos. Rio de Janeiro: Guanabara Koogan, 1996. p. 4-14.

MURPHY, S.; TICKLE-DEGNEN, L. The effectiveness of occupational therapy-related treatments for persons with Parkinson's disease: a meta-analytic review. American Journal Occupational Therapy, Bethesda, v. 55, n. 4, p. 385-392, 2001. http://dx.doi.org/10.5014/ ajot.55.4.385. PMid:11723982.

NICKEL, R. et al. Estudo descritivo do desempenho ocupacional do sujeito com doença de Parkinson: o uso da CIF como ferramenta para classificação da atividade e participação. Revista Acta Fisiátrica, São Paulo, v. 17, n. 1, p. 13-17, 2010.

NUNES, C. M. P. A terapia ocupacional e as disfunções neurológicas da idade adulta e da velhice: CIF em casos clínicos. In: DRUMMOND, A. F.; REZENDE, M. B. (Org.). Intervençōes da Terapia Ocupacional. Belo Horizonte: Editora UFMG, 2008. p. 95-137.

PARKINSON DISEASE FOUNDATION - PDF. New York, 2015. Disponível em: <http://www.pdf. org/>. Acesso em: 23 fev. 2015.

RANSMAYR, G. Physical, occupational, speech and swallowing therapies and physical exercise in Parkinson's disease. Journal Neural Transmission, Wien, v. 118, n. 5, p. 773-781, 2011. http://dx.doi.org/10.1007/s00702011-0622-9. PMid:21461962.

RAO, A. K. Enabling functional independence in Parkinson's disease: update on occupational therapy intervention. Movement Disorders, New York, v. 25, p. 146-151, 2010. Suplemento 1.

RIBERTO, M. et al. Validação da versão brasileira da Medida de Independência Funcional. Revista Acta Fisiátrica, Sáo Paulo, v. 11, n. 2, p. 72-76, 2004.

SHENKMAN, M. L. et al. Spinal movement and performance of standing reach task in participants with and without Parkinson disease. Physical Therapy, Albany, v. 81, n. 8, p. 1400-1411, 2001.

STURKENBOOM, I. H. et al. Effectiveness of occupational therapy in Parkinson's disease: study protocol for a randomized controlled trial. Trials, London, v. 14, p. 1-8, 2013a. PMid:23374761.

STURKENBOOM, I. H. et al. The impact of occupational therapy in Parkinson's disease: a randomized controlled feasibility study. Clinical Rehabilitation, London, v. 27, n. 2, p. 99-112, 2013b. http://dx.doi. org/10.1177/0269215512448382. PMid:22811447.

STURKENBOOM, I. H. et al. Efficacy of occupational therapy for patients with Parkinson's disease: a randomized controlled trial. The Lancet Neurology, London, v. 13, n. 6, p. 557-566, 2014. http://dx.doi.org/10.1016/ S1474-4422(14)70055-9. PMid:24726066.

\section{Contribuiçãa dos Autores}

Todos os autores participaram da concepção, elaboração e redação do artigo e aprovaram a versão final do texto. 